\title{
Vlasov simulations of electron acceleration by radio frequency heating near the upper hybrid layer
}

\author{
A. Najmi ${ }^{1}$, B. Eliasson ${ }^{2}$, X. Shao ${ }^{1}$, G. Milikh ${ }^{1}$, A.S. Sharma ${ }^{1}$, and K. Papadopoulos ${ }^{1}$ \\ ${ }^{1}$ Departments of Physics and Astronomy, \\ University of Maryland, College Park, MD, USA \\ ${ }^{2} S U P A$, Department of Physics, University of Strathclyde, Glasgow, Scotland, UK
}

\begin{abstract}
It is shown by using a combination of Vlasov and test particles simulations that the electron distribution function resulting from energization due to Upper Hybrid (UH) plasma turbulence depends critically on the closeness of the pump wave to the double resonance, defined as $\omega \approx \omega \cup \mathrm{\sim} \approx \omega_{\text {ce }}$ where $\mathrm{n}$ is an integer. For pump frequencies, away from the double resonance the electron distribution function is very close to Maxwellian, while as the pump frequency approaches the double resonance it develops a high energy tail. The simulations show turbulence involving coupling between Lower Hybrid (LH) and UH waves, followed by excitation of Electron Bernstein (EB) modes. For the particular case of a pump with frequency between $n=3$ and $n=4$ the EB modes cover the range from the first to the $5^{\text {th }}$ mode. The simulations show that when the injected wave frequency is between the $3^{\text {rd }}$ and $4^{\text {th }}$ electron cyclotron frequency, bulk electron heating occurs due to the interaction between the electrons and large amplitude EB waves, primarily on the first EB branch leading to an essentially thermal distribution. On the other hand, when the frequency is slightly above the $4^{\text {th }}$ electron cyclotron harmonic, the resonant interaction is predominantly due to the UH branch and leads to a further acceleration of high-velocity electrons and a distribution function with a suprathermal tail of energetic electrons. The results are consistent with ionospheric experiments and relevant to the production of Artificial Ionospheric Plasma Layers.
\end{abstract}

\section{INTRODUCTION}

The coupling of electromagnetic (EM) waves to lower hybrid (LH) and electron Bernstein (EB) waves play important role in different plasma systems, including ionospheric heating experiments Grach et al. (2016), Gurevich (2007) magnetic confinement fusion plasmas Shevchenko et al. (2007), and laboratory plasmas Stenzel and Gould (1971). The UH and EB oscillations are related to the electron cyclotron motion and can efficiently transfer energy to the plasma electrons 
Timofeev (1989), Lominadze (1981). Recent ionospheric heating experiments using ground-based radio-frequency transmitters revealed new features of the turbulence and plasma heating near the UH layer. In particular the HAARP facility at Gakona, Alaska operating at up to $5 \mathrm{GW}$ effective radiated power (ERP) revealed unexpected aspects of wave coupling, plasma turbulence and plasma heating in the high-latitude ionosphere, including artificial ionization and the formation of descending artificial ionospheric layers (DAILs) in the ionospheric F-region Pedersen et al. (2009, 2010). This new feature has been attributed to energetic electrons generated by HF driven ionospheric turbulence. Mishin and Pedersen (2011) introduced an ionizing wave model, in which the energetic electrons ionize the neutrals that form a new interaction region at a progressively lower altitude corresponding to the $\mathrm{O}$ mode reflection altitude. Subsequent models (Eliasson et al., 2012; Mishin et al., 2016) presented detailed numerical model of DAILs. The turbulence at the UH layer is associated with small-scale magnetic field aligned cavities, or density striations (Gurevich, 2007), where a large part of the O mode is absorbed (Mjølhus, 1985, 1988; Eliasson and Papadopoulos, 2015). At the UH resonance layer the Ohmic heating plays an essential role in the formation of density depletions (Dysthe et al., 1982; Jones et al., 1984; Gurevich et al., 1995). At higher pump levels another electron heating mechanism, stochastic heating, becomes effective when the pump wave exceeds a threshold amplitude (Fukuyama et al., 1977; Karney et al., 1978; Balikhin et al.,1993). The stochastic heating is strongly dependent on the nature of the resonances between the electrons and the waves. Previous Vlasov simulations of the HF interaction with the field aligned cavities have shown parametric excitation of UH and EB wave turbulence and stochastic bulk heating of electrons reaching temperatures of 4000-6000 K (Najmi et al., 2016). This mechanism is a part of broader models (Mishin and Pedersen, 2011; Eliasson et al., 2012) for the formation of DAILs based on Strong Langmuir Turbulence (SLT) acceleration at the reflection region by the interaction of electrons heated at the UH layer streaming along the magnetic field lines to the critical layer and are accelerated by SLT to form high-energy suprathermal tails that then could ionize the neutral gas to form new plasma (Eliasson et al., 2012). While the theory and the simulations could explain most of the experimental data a major puzzle remained. The theory based on bulk heating could not account for the so called "double resonance" effect (Bernhardt et al., 2016), viz. the experimental observations that strength of the artificial ionization increases dramatically when the pump frequency $\omega \approx \omega \cup \sim \sim n \omega_{c e}$ where $n$ is an integer. The "double resonance" puzzle can be explained by the fact that the efficiency of the SLT acceleration, being 
a transit type acceleration, depends critically on the percentage of high energy electrons (Mishin et al., 2004, 2016; Papadopoulos et al., 2013). For example, an injected non-thermal distribution function with a significant fraction of suprathermal electrons accelerates much more efficiently than a thermal distribution with the same average energy.

It is the objective of the present work to examine in detail the dependence of the electron distribution function generated by UH/EB turbulence as a function of the closeness of the pump frequency to the double resonance by using a combination of Vlasov and test particle simulations. In particular we investigate the difference in electron heating vs. acceleration for two pump frequencies; a pump frequency close to the $4^{\text {th }}$ as compared to a pump frequency between the $3^{\text {rd }}$ and $4^{\text {th }}$ cyclotron harmonic. The paper is structured as following: In Section II, we present the results of the Vlasov simulations for the two selected frequencies, analyze the resultant frequency spectra and compare with HAARP experiments. In Section III we use test particle simulations to analyze the contributions to the heating of different wave modes. Finally, the results are summarized and discussed in Section V.

\section{VLASOV SIMULATIONS}

Large amplitude UH and EB waves interact strongly with the plasma electrons, and a proper study requires a kinetic description. The Vlasov model, based on the evolution of the distribution function in physical space, velocity space and time, describes wave-particle interactions adequately in most situations where collisions between the charged particles and the neutral gas can be neglected. The Vlasov simulations, with very low noise levels, are well suited for problems involving mode conversion processes and parametric instabilities.

\section{A. Numerical setup}

The evolution of the distribution function $f_{\alpha}$ of charged particles of species $\alpha$ is governed by the Vlasov equation

$$
\frac{\partial f_{\alpha}}{\partial t}+\mathbf{v} \cdot \boldsymbol{\nabla} f_{\alpha}+\frac{\mathbf{F}_{\alpha}}{m_{\alpha}} \cdot \nabla_{\mathbf{v}} f_{\alpha}=0
$$


where the Lorentz force given by

$$
\mathbf{F}_{\alpha}=q_{\alpha}\left(\mathbf{E}+\mathbf{E}_{\text {ext }}+\mathbf{v} \times \mathbf{B}_{0}\right),
$$

and $q_{\alpha}$ and $m_{\alpha}$ are the charge and mass for electrons and ions with $q_{e}=-e, q_{i}=+e$. In UH turbulence the fluctuations are dominantly electrostatic, and consequently in Eq. (2) only the electrostatic field $\mathbf{E}=\boldsymbol{\nabla} \varphi$ is considered, with the electrostatic potential $\varphi$ obtained from the Poisson equation $-\nabla^{2} \varphi=\left(e / \epsilon_{0}\right)\left(n_{i}-n_{e}\right)$. The charge densities are obtained by integrating the distribution functions over velocity space, $n_{\alpha}=\iint f_{\alpha} d v_{x} d v_{y}$.

A simulation code based on Fourier transform in velocity and direct integration in space of the Vlasov equation Eliasson (2010) is used in the numerical simulations in one spatial and two velocity dimensions. In the simulations, we use typical HAARP parameters. The background magnetic field is along the $z$-direction, $\mathbf{B}=\hat{\mathbf{z}} B_{0}$, and the spatial variations are taken to be along the $x$-direction, perpendicular to the magnetic field. The amplitude of the magnetic field is $B_{0}=$ $5.17 \times 10^{-5} \mathrm{~T}$, corresponding to the electron cyclotron frequency $\omega_{c e}=9.09 \times 10^{6} \mathrm{~s}^{-1}\left(f_{c e}=\right.$ $1.45 \mathrm{MHz}$ ). The incident $\mathrm{O}$ mode electromagnetic wave is represented by an external oscillating dipole electric field, $\mathbf{E}_{\text {ext }}=\hat{\mathbf{x}} E_{0} \sin \left(\omega_{0} t\right)$ with amplitude $E_{0}=2.0 \mathrm{~V} / \mathrm{m}$. The ions provide the neutralizing background and play a significant role through fluctuations near the LH frequency. Although the dominant ion species near the UH layer in the ionosphere is atomic oxygen, we use the proton-to-electron mass ratio, $m_{i} / m_{e}=1836$ for numerical efficiency. The spatial domain of width $9 \mathrm{~m}$ is resolved into a grid of 600 elements, resulting into a spatial grid-size of $1.5 \mathrm{~cm}$. Periodic boundary conditions are used in space. The standard $4^{\text {th }}$-order Runge-Kutta method is used to advance the solution in time, with the time step chosen dynamically to maintain numerical stability, using a typical value of the time-step of $7.0 \mathrm{~ns}$. The velocity variable $\mathbf{v}$ has the two components $v_{x}$ and $v_{y}$ directed in the $x-y$ plane, perpendicular to the magnetic field direction. The electrons have a velocity grid spacing of $0.31 v_{T e 0}$ while the ions have a velocity grid of $0.22 v_{T i 0}$ in both $v_{x}$ and $v_{y}$. We use initial temperatures $T_{e 0}=1500 \mathrm{~K}$ for electrons and $T_{i 0}=1000 \mathrm{~K}$ for ions, corresponding to the electron and ion thermal speeds $v_{T e 0}=1.51 \times 10^{5} \mathrm{~m} / \mathrm{s}$ and $v_{T i 0}=$ $2.87 \times 10^{3} \mathrm{~m} / \mathrm{s}$.

The initial electron and ion number density is taken as a superposition of the ambient density $n_{0}$ and a density depletion (striation) with a Gaussian profile, given by 


$$
n_{i}(x)=n_{e}(x)=n_{0}\left[1-\alpha \exp \left(-\frac{x^{2}}{R^{2}}\right)\right],
$$

with the transverse scale $R=1.5 \mathrm{~m}$ and normalized amplitude $\alpha=0.1$. In addition to the transverse profile of the density striation, the vertical stratification of the ionosphere leads to a gradual increase of the plasma density with altitude and a finite width of the UH layer where the O mode can be converted to UH waves in the striations Mjølhus (1985, 1988), Eliasson and Papadopoulos (2015). We consider two cases of the HF waves with different frequencies, $\omega_{0}=$

$3.80 \omega_{c e}=3.45 \times 10^{7} \mathrm{~s}^{-1}\left(f_{0}=5.50 \mathrm{MHz}\right)$ and $\omega_{0}=4.01 \omega_{c e}=3.65 \times 10^{7} \mathrm{~s}^{-1}\left(f_{0}=5.80\right.$ $\mathrm{MHz}$ ). For the two frequency cases, the ambient number densities outside the striation are taken to be $n_{0}=3.67 \times 10^{11} \mathrm{~m}^{-3}$ and $4.12 \times 10^{11} \mathrm{~m}^{-3}$, respectively, giving the corresponding electron plasma frequencies $\omega_{p e}=3.42 \times 10^{7} \mathrm{~s}^{-1}$ and $3.62 \times 10^{7} \mathrm{~s}^{-1}\left(f_{p e}=5.44 \mathrm{MHz}\right.$ and $5.77 \mathrm{MHz}$ ). The two pump frequencies are chosen to maintain $\omega_{0}=\omega_{U H}$ at $0.95 n_{0}$ as $\omega_{0}$ is varied through the gyro-harmonics. The corresponding LH frequencies are $f_{L H}=32.64 \mathrm{kHz}$ and $32.76 \mathrm{kHz}$, respectively.

\section{B. Simulation results of electron heating and acceleration}

Here we discuss the results of the Vlasov and test-particle simulations at the two different pump frequencies $\omega_{0}=3.80 \omega_{c e}$ and $\omega_{0}=4.01 \omega_{c e}$ for pump amplitude $2.0 \mathrm{~V} / \mathrm{m}$ expected for HAARP injection Eliasson et al. (2012). 

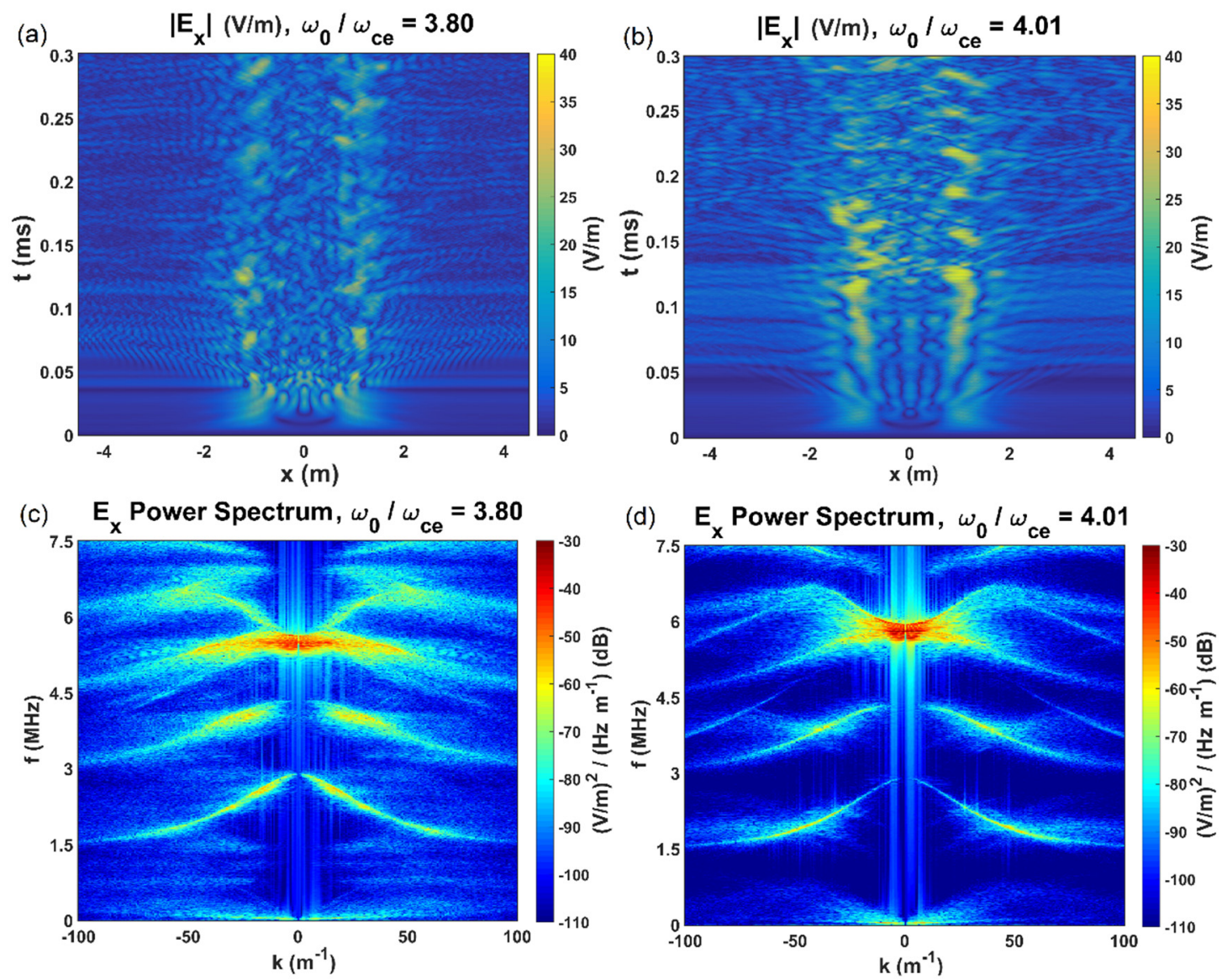

FIG. 1. Top panels: Evolution of the electric field using (a) $\omega_{0}=3.80 \omega_{c e}\left(f_{0}=5.50 \mathrm{MHz}\right)$ and (b) $\omega_{0}=4.01 \omega_{c e}\left(f_{0}=5.80 \mathrm{MHz}\right)$. Bottom panels: Frequency-wavenumber power spectra of the electric field using (c) $\omega_{0}=3.80 \omega_{c e}$ and (d) $\omega_{0}=4.01 \omega_{c e}$.

Plots of the time evolution of the electric field and the corresponding frequency-wavenumber power spectra are shown in Fig. 1. The pump wave of amplitude $2.0 \mathrm{~V} / \mathrm{m}$ is mode converted to trapped electrostatic UH oscillations with amplitudes $\sim 40 \mathrm{~V} / \mathrm{m}$, seen in Figs. 1a and 1b. The spectra in the two cases, shown in Figs. 1c and 1d, are qualitatively similar. Several branches of EB waves are visible, with frequencies that asymptotically approach resonances at the electron cyclotron harmonics, viz. $f_{E B}=1.5,3.0,4.5$, and $6.0 \mathrm{MHz}$, at large wavenumbers. In both cases, the high amplitude pump frequencies between $5.50-5.80 \mathrm{MHz}$ and near $k=0$ dominate the power spectra. EB waves near UH frequency is also excited at larger wavenumbers extending out to $k=$ $50 \mathrm{~m}^{-1}$ for $\omega_{0}=3.80 \omega_{c e}$ (Fig. 1c) and to $k=20 \mathrm{~m}^{-1}$ for $\omega_{0}=4.01 \omega_{c e}$ (Fig. 1d). The LH waves mirror a similar behavior at $f \approx 30 \mathrm{kHz}$. 

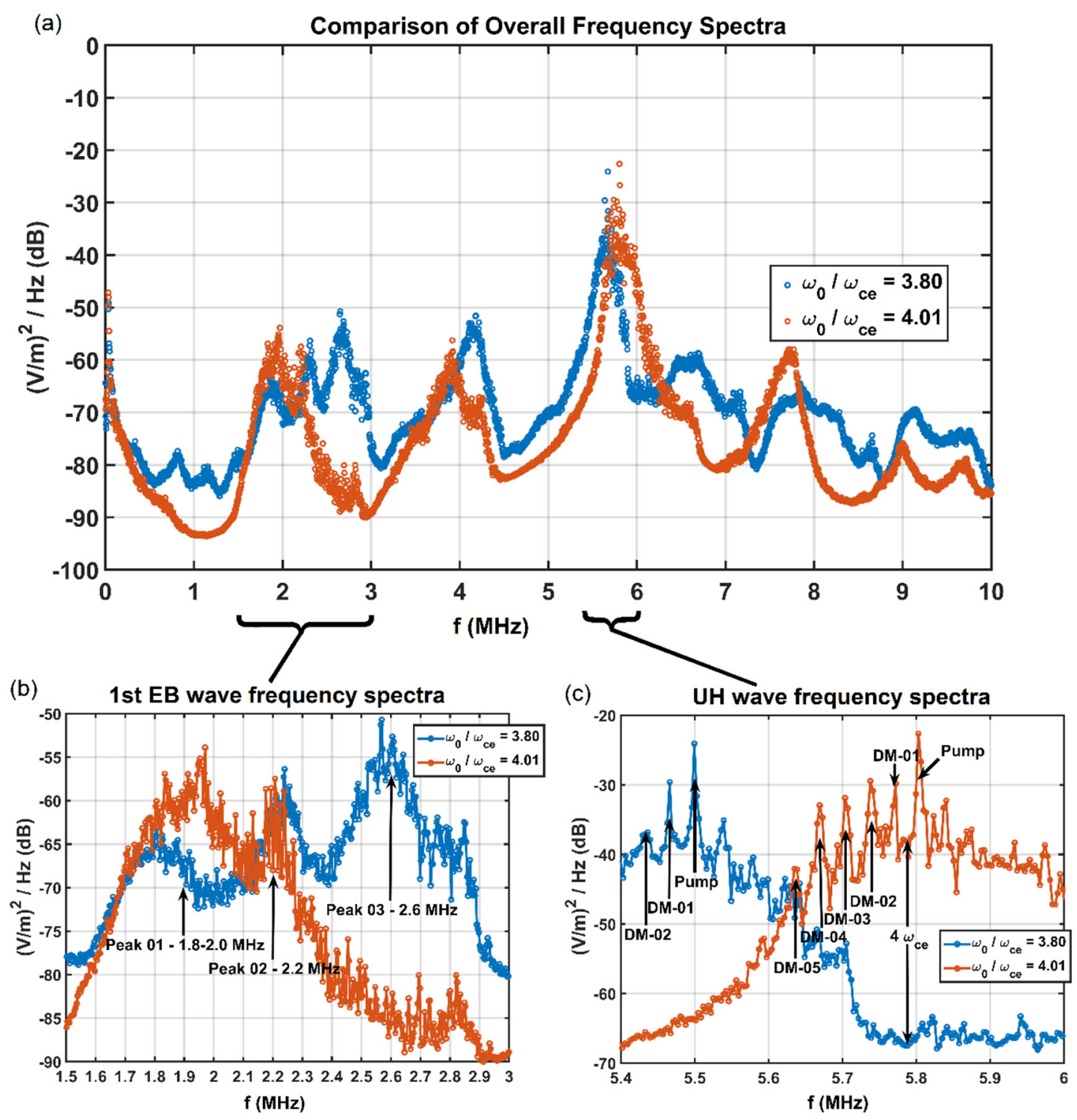

FIG. 2. Comparison of frequency spectra for $\omega_{0} / \omega_{c e}=3.80$ and 4.01. (a) The overall frequency ranges from $0-10 \mathrm{MHz}$, (b) a zoom in at $1.5-3.0 \mathrm{MHz}$ corresponding to the first EB wave and (c) a zoom-in at 5.4-6.0 MHz covering the $\mathrm{UH}$ frequency.

The frequency spectra, obtained by integrating the frequency-wavenumber power spectra in Fig. 1 over wavenumber space, are shown in Fig. 2. The overall frequency spectra shown in Fig. 2a, while a close-up of the wave spectra around the $1^{\text {st }} \mathrm{EB}$ branch is shown in Fig. $2 \mathrm{~b}$ in the frequency interval $1.5-3 \mathrm{MHz}$, and a close-up around the UH resonances is shown in Fig. 2c in the frequency interval 5.4 - $6 \mathrm{MHz}$. As seen in Fig. 2b, there are peaks near 1.8, 2.2, and 2.6 MHz for the case of $\omega_{0} / \omega_{c e}=3.80$, while for $\omega_{0} / \omega_{c e}=4.01$, there are peaks only at 1.8 and $2.2 \mathrm{MHz}$ but not at 2.6 
MHz. In Fig. 2c, we have indicated the pump frequency and several downshifted maxima (DM) and an upshifted maximum (UM) which are separated from the pump by multiples of the LH frequency, and the location of the $4^{\text {th }}$ electron cyclotron harmonic, $4 f_{c e}=5.79 \mathrm{MHz}$ is also indicated.

\section{Comparison with Experiments}
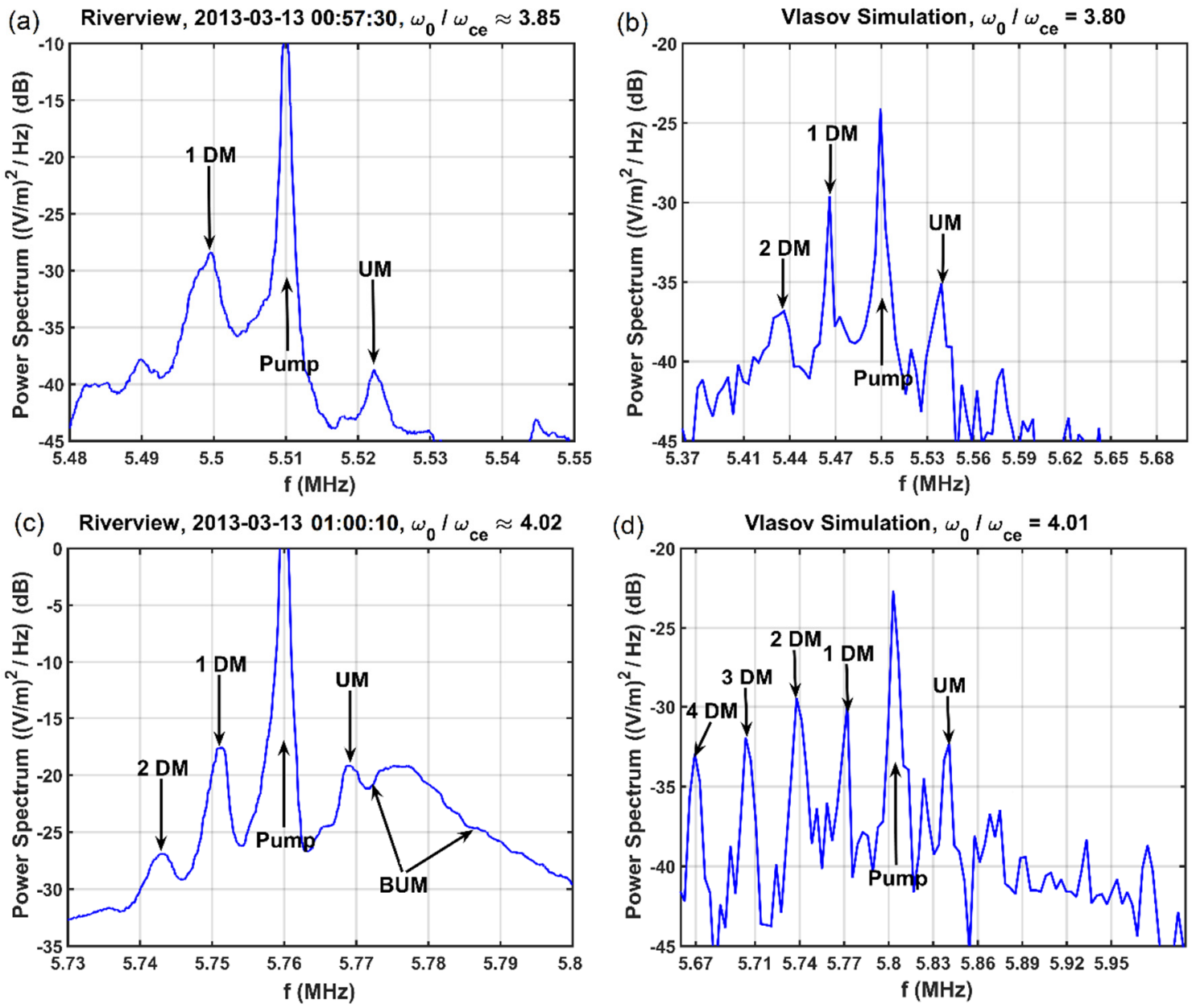

FIG. 3. Comparison of stimulated electromagnetic emissions (SEE) recorded by ground-based detectors during an ionospheric heating experiment [panels (a), (c)], with Vlasov simulation results using similar pump frequencies [panels (b), (d)]. The downshifted maxima (DM) and upshifted maxima (UM) are present in the two cases, but the broad upshifted maximum (BUM) is seen only for $\omega_{0} / \omega_{c e} \approx 4.02$ in the experiment [panels (c)].

To assess the validity of the Vlasov simulations, comparisons of frequency spectra obtained in an ionospheric heating experiment is presented in Fig. 3. The experiment was carried out during the March 2013 campaign at the HAARP facility by stepping an O-mode pump wave in frequency from $300 \mathrm{kHz}$ below to $200 \mathrm{kHz}$ above the $4^{\text {th }}$ electron cyclotron harmonic, while simultaneously 
remaining below the F2 critical layer to be near the UH resonance. Details of the experimental setup is given by Najmi et al. (2014). Ground-based instruments operated by the Naval Research Laboratory (NRL) recorded Stimulated Electromagnetic Emissions (SEE) during the experiments. As seen in Fig. 3, the spectra obtained from the SEE measurements show similar features as the electric field spectra from our Vlasov simulations at similar pump frequencies. In the F-region, the dominant ion species is atomic oxygen, with an atomic mass 16 times higher than the proton mass used in our simulations, and has a corresponding LH frequency of $1 / \sqrt{16}=1 / 4$ of our simulation value of $\approx 32 \mathrm{kHz}$. Therefore, while our simulations show a separation between the pump and the downshifted maximum $(\mathrm{DM})$ of $\approx 32 \mathrm{kHz}$, the NRL data shows a separation of $\approx 8 \mathrm{kHz}$, as expected. For the cases using a pump frequency between the $3^{\text {rd }}$ and $4^{\text {th }}$ electron cyclotron harmonic, shown in Figs. 3a and 3b, the pump wave is clearly visible, as well as a DM separated from the pump by appropriately the LH frequency, and an upshifted maximum (UM) that is upshifted by a similar amount. In the cases using a pump frequency slightly above the $4^{\text {th }}$ cyclotron harmonic, shown in Figs. 3c and 3d, additional DMs are seen, and there is also a broad upshifted maximum (BUM) associated with the $4^{\text {th }} \mathrm{EB}$ wave. While the BUM is formed for $\omega_{0} / \omega_{c e}=4.02$, it is absent for $\omega_{0} / \omega_{c e}=3.85$ in the SEE observations in Fig. 3c. The absence of the BUM in the simulated spectrum in Fig. $3 \mathrm{~d}$ is consistent with that a finite angle of the LH waves to the magnetic field is required for the BUM to form Huang and Kuo (1994), Xi and Scales (2001), while the twodimensional (2D) Vlasov simulations support only perpendicular propagation. It should be noted that while the UH turbulence has frequencies above the local plasma frequency so that the $\mathrm{O}$ mode wave can couple to the turbulence and escape the plasma to produce SEE, oscillations on the lower EB modes seen in the simulations and shown in Fig. 2 have frequencies below the local plasma frequency and hence would require in-situ measurements to be observed.

\section{TEST PARTICLE SIMULATIONS}

The plasma turbulence excited by the HF heating consists of HF wave modes associated with the electrons coupled with low-frequency modes involving also the ions. The analysis of the physical mechanisms underlying the electron bulk heating and acceleration to suprathermal tails requires details of the electron dynamics in the turbulent electric fields. Test particle simulations are carried out using the equations of motion of the electrons accelerated by the Lorenz force given by Eq. (2), with the wave electric field obtained from the Vlasov simulations. The test particles are 
accelerated by the given electric field, but are not assumed themselves to contribute to the electric field. The particle positions are initialized within the Vlasov simulation domain, and the particle velocities are randomly initialized to a 2D Maxwellian distribution with temperature $T_{e 0}$.

\section{A. Heated and resonantly accelerated electrons by different wave modes}
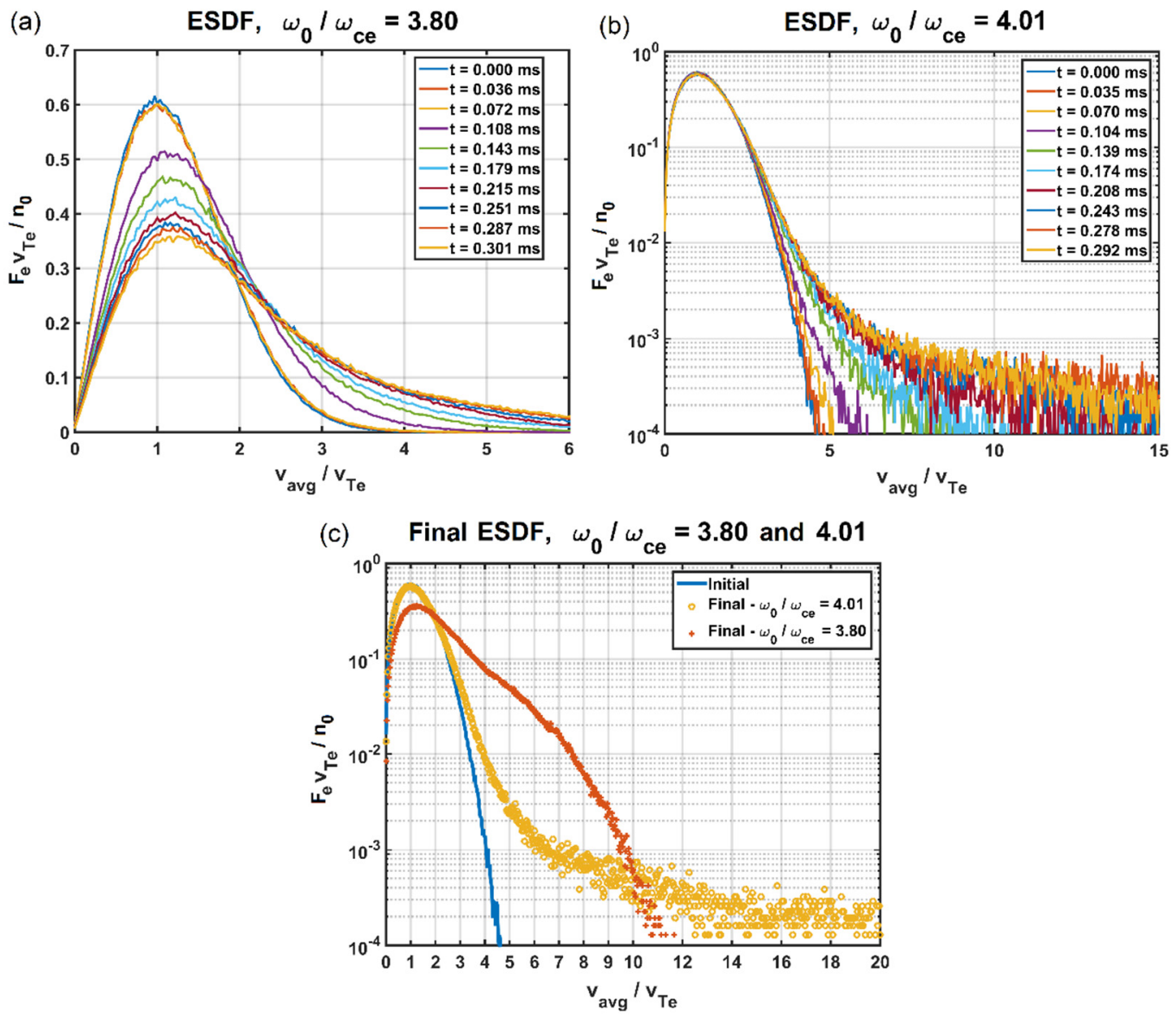

FIG. 4. Evolution of average electron speed distribution for (a) $\omega_{0} / \omega_{c e}=3.80$, using a linear scale to illustrate the bulk heating leading to a widening of the electron speed distribution function, and (b) $\omega_{0} / \omega_{c e}=4.01$, on a logarithmic scale to illustrate the suprathermal tail at high speeds. (c) Initial and final distribution functions from both pump frequencies using a log-scale.

Plots of the electron speed distribution functions from the test particle simulations are shown in Fig. 4. The electron speed distribution function (ESDF) is obtained from the 2D electron distribution function by assuming that it is gyrotropic, $f_{e}\left(v_{x}, v_{y}\right)=f_{e}(v)$, where $v=\sqrt{v_{x}^{2}+v_{y}^{2}}$ is the speed. Using the polar coordinates $v_{x}=v \cos \phi$ and $v_{y}=v \sin \phi$ and integrating over $\phi$ gives the differential electron number density $d n_{e}=2 \pi f_{e}(v) v d v \equiv F_{e}(v) d v$, with $F_{e}(v)=$ $2 \pi f_{e}(v) v$ being the ESDF, and the electron number density is obtained as $n_{e}=\int_{0}^{\infty} F_{e} d v$. 
Figures $4 \mathrm{a}$ and $4 \mathrm{~b}$ show the ESDF as a function of $v=v_{\text {avg }}$ at various times, where $v_{\text {avg }}$ is the particle's speed, averaged over a pump wave period. For the pump frequency $\omega_{0}=3.80 \omega_{c e}$, we observe in Fig. 4a bulk heating of the electrons, leading to a spread of the ESDF as a function of $v_{a v g}$. For $\omega_{0}=4.01 \omega_{c e}$ in Fig. $4 \mathrm{~b}$, only the tail of the energy distribution is accelerated. The final distribution functions in Fig. 4c further illuminate this difference, with bulk heated electrons for $\omega_{0}=3.80 \omega_{c e}$ and the formation of suprathermal tails with no bulk heating for $\omega_{0}=4.01 \omega_{c e}$.
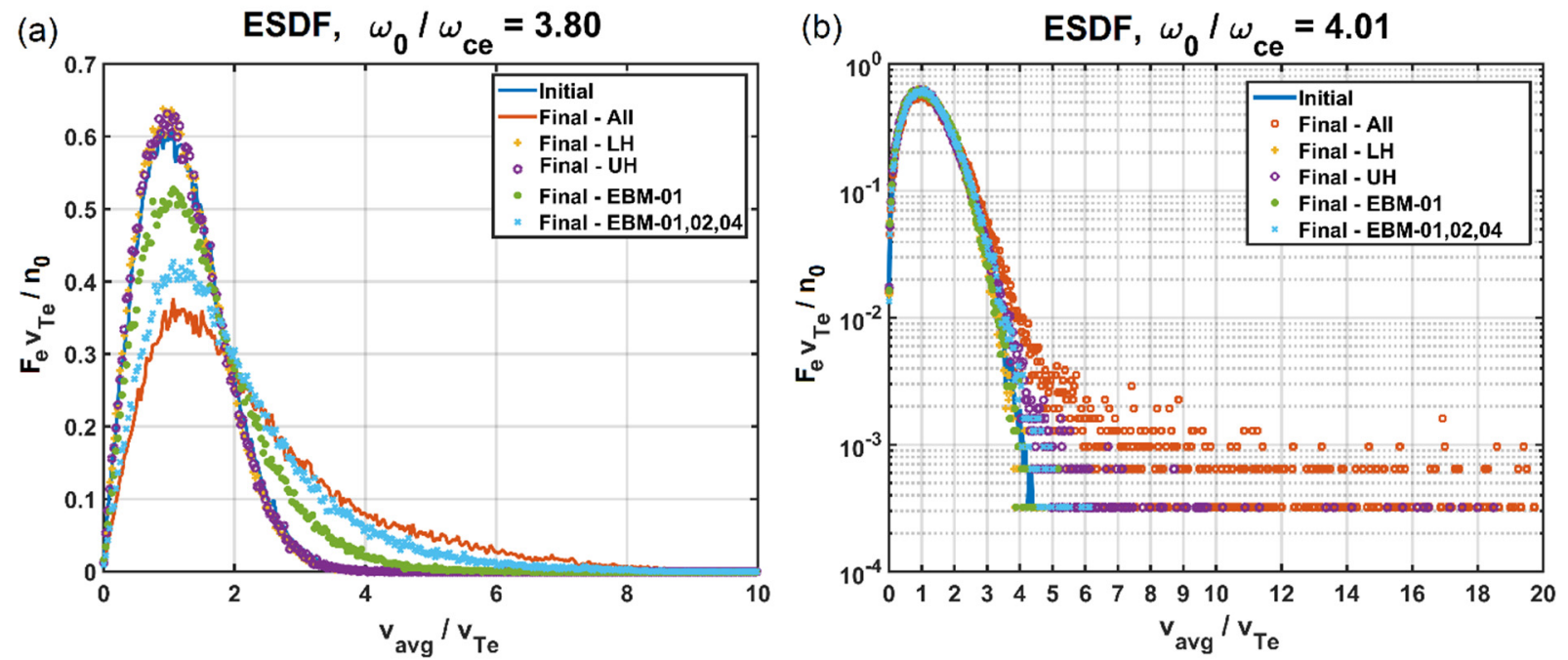

FIG. 5. The averaged electron speed distribution function for one wave mode at the time for (a) $\omega_{0} / \omega_{c e}=3.80$ using a linear scale to illustrate the bulk heating leading to a widening of the electron speed distribution function, and (b) $\omega_{0} / \omega_{c e}=4.01$ on a logarithmic scale to illustrate the suprathermal tail at high speeds.

By filtering all but a single wave mode at a time, we study which effect each individual wave mode has on the electron distribution functions. The results are shown in Fig. 5, where we include plots of the initial and final ESDF with all wave modes, as well as the final ESDF with isolated LH, UH, and EB waves. For the pump frequency $\omega_{0}=3.80 \omega_{c e}$ (Fig. 5a), the dominant contribution to the bulk heating comes from EB waves, while for $\omega_{0}=4.01 \omega_{c e}$ (Fig. 5b), the contribution to the electron tail heating comes almost entirely from the UH waves. 

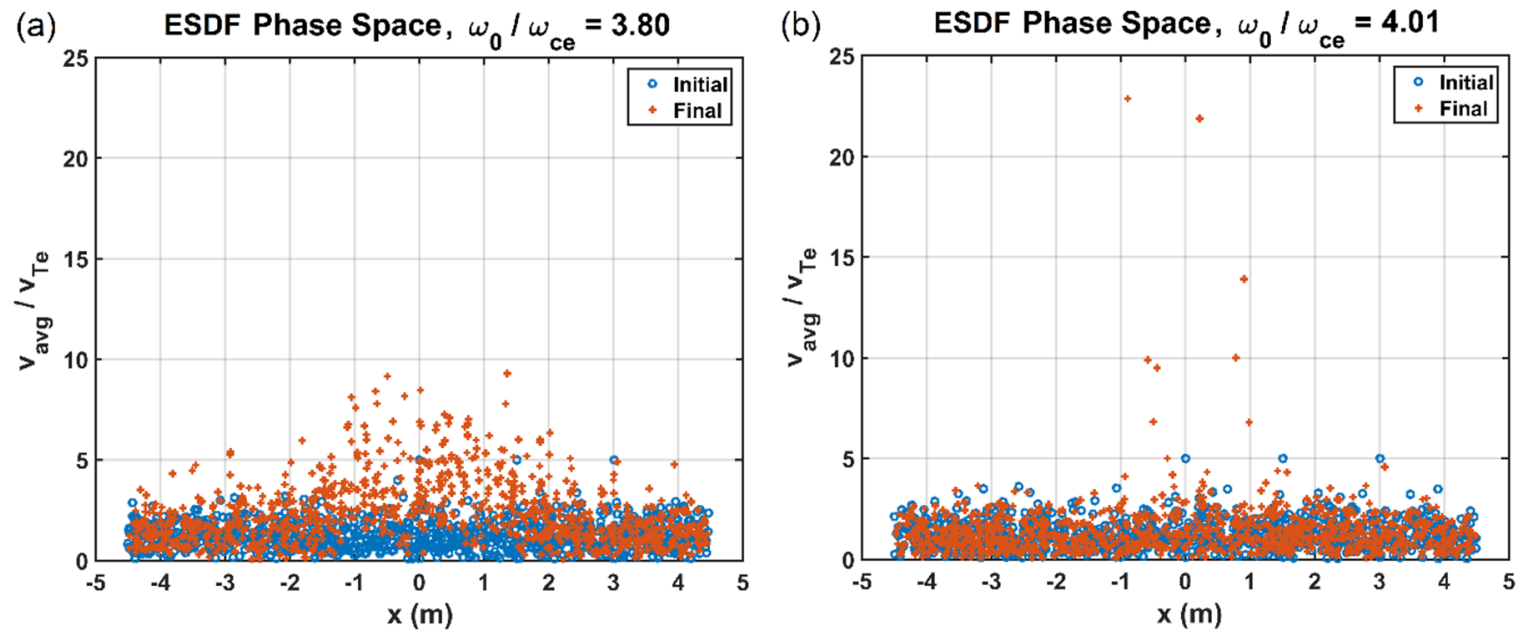

FIG. 6. Initial and final $x-v_{\text {avg }}$ phase space for pump wave (a) with $\omega_{0}=3.80 \omega_{c e}$, and (b) with $\omega_{0}=4.01 \omega_{c e}$. In both cases, particles are accelerated within the striation $(-1.5<x<1.5)$ while particles outside the striation are mostly unperturbed.

It is illuminating to study how particles are accelerated at different positions in space at and around the striation. Figure 6 shows the initial and final $x-v_{\text {avg }}$ phase space of electrons for each of the two pump waves. In both cases, accelerated particles are primarily drawn from those inside the striation $(-1.5 \mathrm{~m}<x<1.5 \mathrm{~m})$. For the case $\omega_{0}=3.80 \omega_{c e}$, shown in Fig. 6a, particles initially occupying phase space in the region $-1.5 \mathrm{~m}<x<1.5 \mathrm{~m}$ and $0<v_{\text {avg }}<2 v_{T e}$ have been pushed up into $2 v_{T e}<v_{\text {avg }}<7 v_{T e}$, while particles outside the striation or with initial velocity greater than about 3-5 $v_{T e}$ experience little or no acceleration. For $\omega_{0}=4.01 \omega_{c e}$ in Fig. $6 \mathrm{~b}$, there is a small number of fast particles that are further accelerated. Particles with small initial velocities are not accelerated, independent of their location in the striation. 

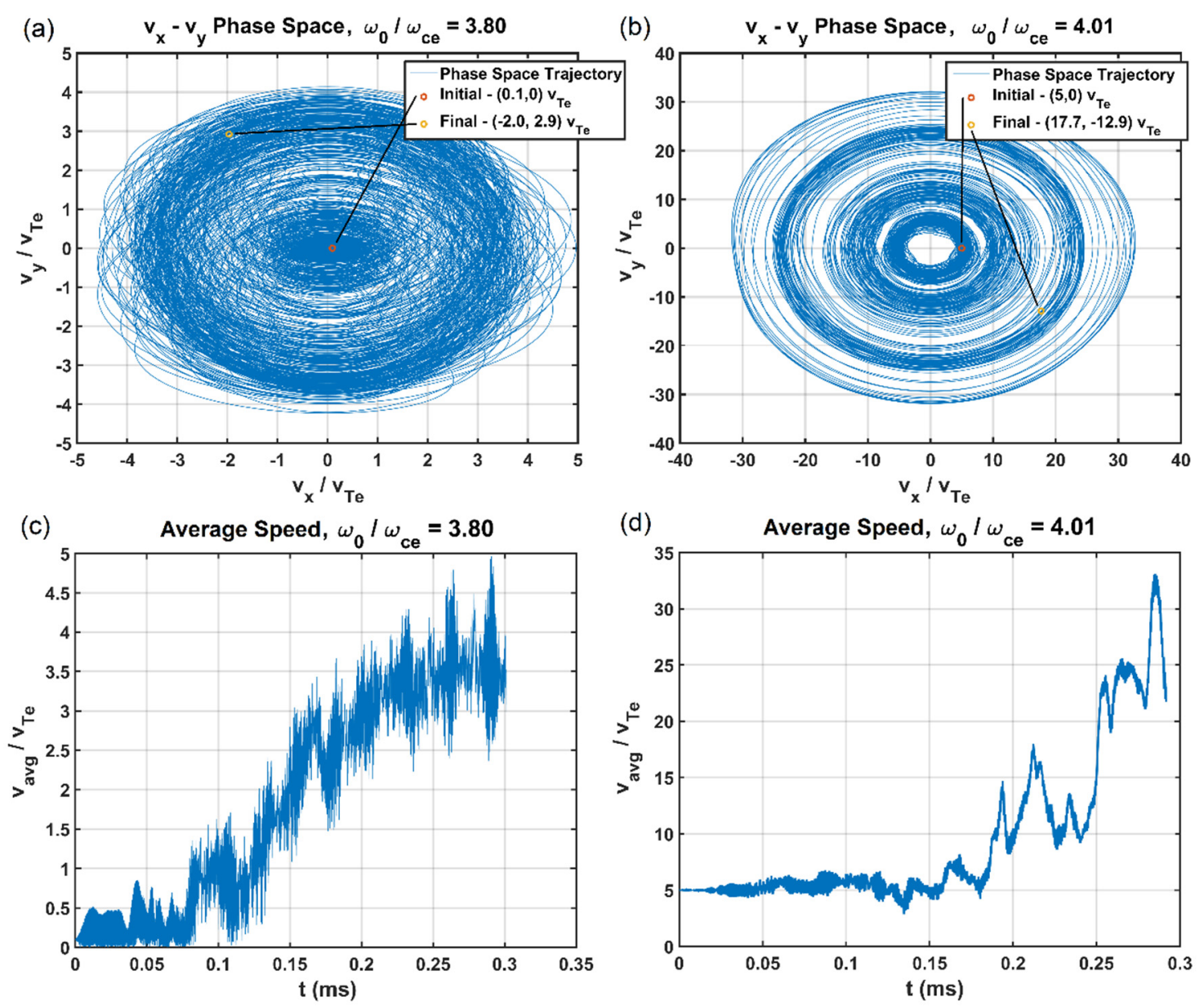

FIG. 7. Phase space trajectories and time-dependence of $v_{\text {avg }}$ for a test particle for the cases $\omega_{0}=$ $3.80 \omega_{c e}$ and $\omega_{0}=4.01 \omega_{c e}$. In (a) and (c) an initially slow particle on the edge of the striation using $\omega_{0}=3.80 \omega_{c e}$, is steadily heated to several times $v_{T e}$ after an onset time of $\approx 0.07 \mathrm{~ms}$. In (b) and (d), an initially fast particle is resonantly accelerated to over $20 v_{T e}$ in discrete steps that occur after an onset time of $\approx 0.15 \mathrm{~ms}$.

In Fig. 7, we examine in more detail the case of a sample particle to elucidate the nature of the energization for the cases $\omega_{0}=3.80 \omega_{c e}$ and $\omega_{0}=4.01 \omega_{c e}$. The $v_{x}-v_{y}$ phase space trajectories in the two cases for a selected particle are shown in Figs. $7 \mathrm{a}$ and $7 \mathrm{~b}$, and the time dependence of $v_{\text {avg }}$ for each particle is shown in Figs. $7 \mathrm{c}$ and $7 \mathrm{~d}$. These plots show the di erence between the steadier bulk heating of an initially slow particle in the case $\omega_{0}=3.80 \omega_{c e}$, and the discrete "kicks" received by an initially fast particle for $\omega_{0}=4.01 \omega_{c e}$. Figures $7 \mathrm{c}$ and $7 \mathrm{~d}$ show the onset time of acceleration that can be correlated with electric field data. For $\omega_{0} / \omega_{c e}=3.80$, the onset occurs after $\approx 0.07 \mathrm{~ms}$, while for $\omega_{0} / \omega_{c e}=4.01$, the resonant acceleration does not begin until $\approx$ $0.12-0.15 \mathrm{~ms}$. 

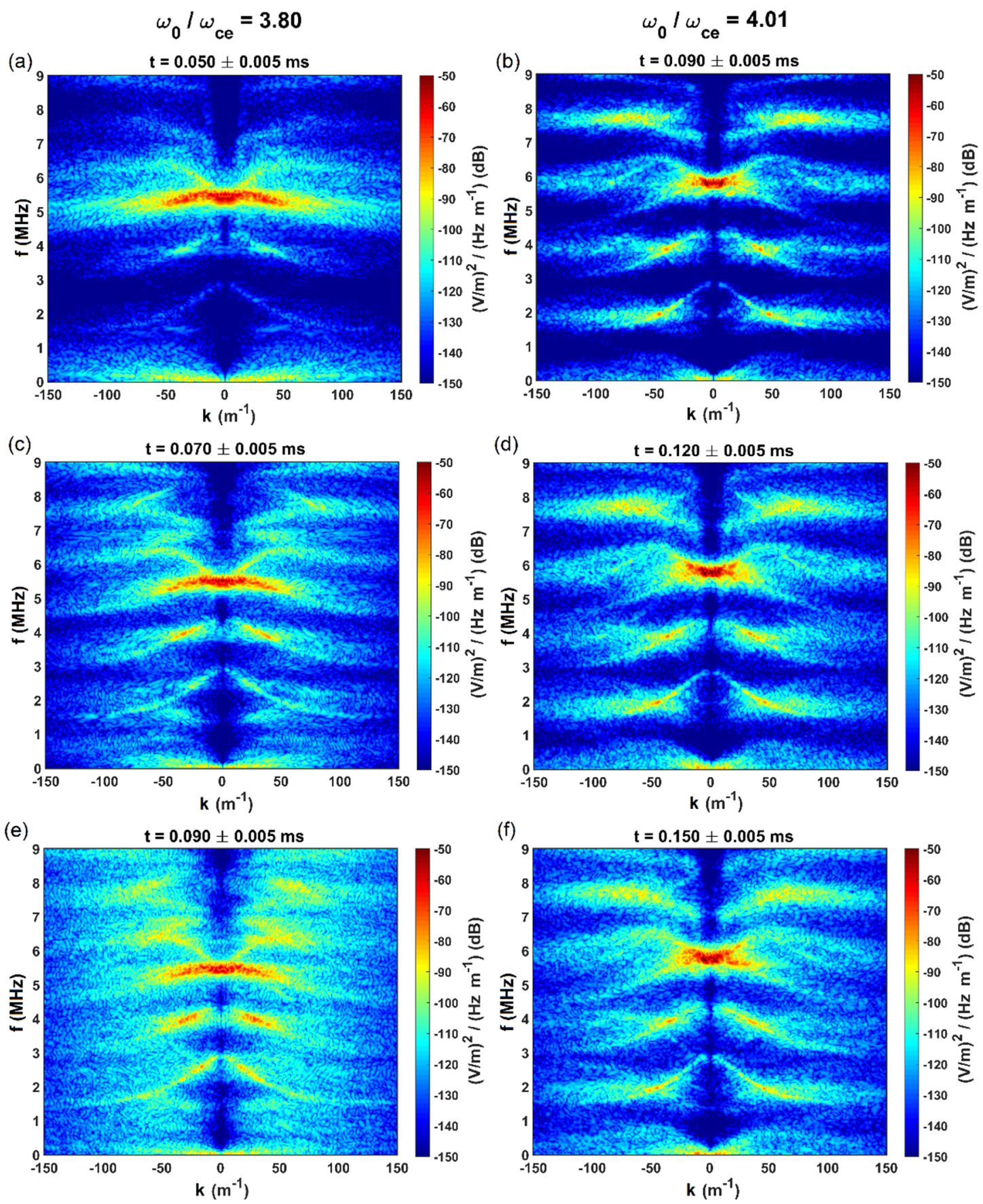

FIG. 8. Frequency-wavenumber power spectra of the electric field, with a Gaussian window centered at $x=0$ using the width $\sigma_{x}=1.0 \mathrm{~m}$, and at time $t$ (indicated above each panel) using the temporal width $\sigma_{t}=0.005 \mathrm{~ms}$. Left column shows spectra for $\omega_{0} / \omega_{c e}=3.80$, and right column show spectra for $\omega_{0} / \omega_{c e}=4.01$. 
In Fig. 8, we show the evolution of the electric field spectra in the neighborhood of the onset times noted in the test-particle simulations above, by taking Fourier transforms of the electric field in space and time after applying a Gaussian window centered on the bottom of the striation, $x=0$, with $\sigma_{x}=1.0 \mathrm{~m}$, and centered at different times using the temporal window $\sigma_{t}=0.005 \mathrm{~ms}$ in each case. In Figs. $8 \mathrm{a}, 8 \mathrm{c}$, and $8 \mathrm{e}$ for $\omega_{0}=3.80 \omega_{c e}$, the first EB wave is absent at $0.05 \mathrm{~ms}$, but starts to develop at $0.07 \mathrm{~ms}$, and is fully formed by $0.09 \mathrm{~ms}$, consistent with the heating onset time of $\approx$ $0.07 \mathrm{~ms}$, and also that the first EB wave is a primary driver of bulk heating. In Figs. $8 \mathrm{~b}, 8 \mathrm{~d}$, and $8 \mathrm{f}$ for $\omega_{0}=4.01 \omega_{c e}$, the UH and LH spectra grow sharply in both amplitude and wavenumber from $0.09 \mathrm{~ms}$, expanding the UH spectrum to nearly $k=50 \mathrm{~m}^{-1}$ at $0.15 \mathrm{~ms}$, consistent with the observed heating onset in test particle simulations of $\approx 0.12-0.15 \mathrm{~ms}$, and also with the UH spectrum near $4 \omega_{c e}$ as the primary cause of tail heating. Ion density fluctuations (not shown) also have distinct onset times, with fully developed LH oscillations from about $0.03 \mathrm{~ms}$ onward for $\omega_{0} / \omega_{c e}=3.80$, and some LH oscillations visible at $0.05 \mathrm{~ms}$, but not fully developed until $\approx$ $0.12-0.15 \mathrm{~ms}$ for $\omega_{0} / \omega_{c e}=4.01$.

\section{B. Excitation of Electron Bernstein (EB) waves}
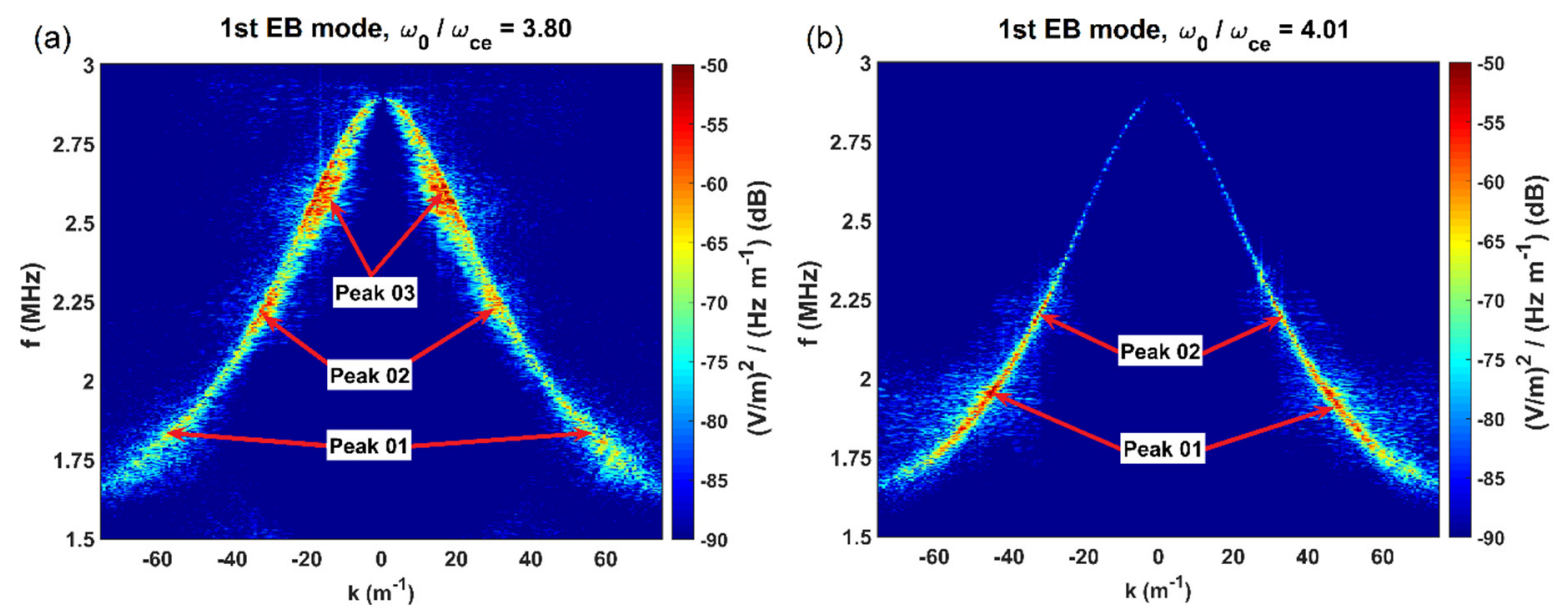

FIG. 9. Frequency-wavenumber power spectra for the first EB wave (a) using $\omega_{0} / \omega_{c e}=3.80$, and (b) using $\omega_{0} / \omega_{c e}=4.01$. The peaks identified in Fig. 2b are as marked. Peak 03 of (a), associated with the bulk heating of electrons, corresponds to a frequency of 2.6-2.7 MHz and a wavenumber of $15-20 \mathrm{~m}^{-1}$.

By carrying out a more detailed study similar to the one in Fig. 5 (not shown), we have identified the most important wave mode associated with bulk heating to be Peak 03 (cf. Figs. 2b and 9a) at 
2.6-2.7 MHz on the first EB wave. In Fig. 9, we show the frequency-wavenumber power spectra of the first EB wave for both pump frequencies. To excite waves at Peak 03, we consider the linear mode conversion of the pump wave $\left(E_{0}, \omega_{0}\right)$ to $\mathrm{UH}$ waves at the edge of the density cavity where $n=0.95 n_{0}$ and a subsequent three-wave decay obeying the frequency and wave number matching conditions

$$
\omega_{0}=\omega_{1}+\omega_{2}
$$

and

$$
k_{0}=k_{1}+k_{2} \text {. }
$$

A direct decay from a mode converted UH pump wave at $\omega_{0}=3.80 \omega_{c e}(5.50 \mathrm{MHz})$ to two EB waves of frequency $1.63-1.69 \times 10^{7} \mathrm{~s}^{-1}(2.6-2.7 \mathrm{MHz}$, see Peak 03 in Fig. 2b) is prohibited by the frequency matching condition. However, if the pump wave first decays to UH and LH waves and then a daughter UH wave decays to two EB waves, the frequency matching condition can be satisfied, and this would also explain the observed onset time of EB waves after the formation of a broad wavenumber spectrum of UH and LH turbulence.

Following the results presented in Najmi et al. (2016), we consider a mode converted UH wave that propagates to the center of the striation and undergoes a three-wave decay to a daughter $\mathrm{UH}$ wave and a LH wave. The frequency di erence between the pump frequency and the local UH frequency at the bottom of the cavity is given by

$$
\Delta \omega=\sqrt{0.95 \omega_{p e}^{2}+\omega_{c e}^{2}}-\sqrt{0.90 \omega_{p e}^{2}+\omega_{c e}^{2}}=8.66 \times 10^{7} \mathrm{~s}^{-1}(136 \mathrm{kHz}) .
$$

The LH frequency is $\omega_{L H}=2.05 \times 10^{5} \mathrm{~s}^{-1}(32.64 \mathrm{kHz})$, and $\Delta \omega / \omega_{L H}=4.18$, giving enough room in frequency space for up to four successive decays of an UH wave to a downshifted UH wave, with each downshift equal to the LH frequency. To find the frequency and wavenumber of the daughter UH waves, we take the dispersion relation for UH waves Lominadze (1981)

$$
\omega^{2}=\omega_{U H}^{2}+\frac{3 v_{T e}^{2} k^{2} \omega_{p e}^{2}}{\omega^{2}-4 \omega_{c e}^{2}}
$$

and substitute both the frequency matching condition (4), and the local UH frequency, assuming that the bottom of the density cavity is homogenous to first order.

The $N$ th daughter UH wave $\left(\omega_{0}-N \omega_{L H}, k_{N}\right)$ has the dispersion relation 


$$
\left(\omega_{0}-N \omega_{L H}\right)^{2}=0.9 \omega_{p e}^{2}+\omega_{c e}^{2}+\frac{3 v_{T e}^{2} k_{N}^{2} \omega_{p e}^{2}}{\left(\omega_{0}-N \omega_{L H}\right)^{2}-4 \omega_{c e}^{2}}
$$

For $N=4$, we have $\omega_{4}=3.37 \times 10^{7} \mathrm{~s}^{-1}(5.37 \mathrm{MHz})$ and $k_{4}=5.12 \mathrm{~m}^{-1}$. The frequency $\omega_{4}$ can satisfy the frequency matching condition for decay to two EB waves with frequencies 1.63$1.69 \times 10^{7} \mathrm{~s}^{-1}(2.6-2.7 \mathrm{MHz})$ since $\omega_{4} / 2=1.69 \times 10^{7} \mathrm{~s}^{-1}(2.68 \mathrm{MHz})$, and the wavenumber $k_{4}$ can satisfy the wavenumber matching condition for waves with $k=15-20 \mathrm{~m}^{-1}$ which are the ranges of the $3^{\text {rd }}$ peak of the first EB wave in Fig. 8a. For $\omega_{0} / \omega_{c e}=4.01$, we can use this same procedure to show that a decay is not possible, since $\Delta \omega / \omega_{L H}=4.43, \omega_{4}=3.56 \times 10^{7} \mathrm{~s}^{-1}(5.67$ $\mathrm{MHz}$ ) is prohibited by the frequency matching condition to decay to two EB waves with frequency $1.63-1.69 \times 10^{7} \mathrm{~s}^{-1}(2.6-2.7 \mathrm{MHz})$.

\section{Analysis of stochastic heating for a plane wave}

We here carry out test particle simulations of the dynamics and collisionless heating of electrons in a static magnetic field $\mathbf{B}_{0}=B_{0} \hat{\mathbf{z}}$, subject to a plane electrostatic wave field of the form $\mathbf{E}=$ $E_{0} \sin \left(k x^{j}-\omega t\right) \hat{\mathbf{x}}$, where $\hat{\mathbf{x}}$ and $\hat{\mathbf{z}}$ are the unit vectors in the $x$ - and $z$-directions. This is a highly idealized model assuming a monochromatic wave, which however can be justified since the electrons are restricted in space perpendicular to the magnetic field within its gyro-radius. The equations of motion for each test-particle, representing an electron, are

$$
m_{e} \frac{d \mathbf{v}^{j}}{d t}=-e E_{0} \sin \left(k x^{j}-\omega t\right) \hat{\mathbf{x}}-e \mathbf{v}^{j} \times \mathrm{B}_{0} \hat{\mathbf{z}},
$$

and

$$
\frac{d x^{j}}{d t}=v_{x}^{j}
$$

where the velocity $\mathbf{v}^{j}$ and position $\mathbf{x}^{j}$ depend on time $t$ for each particle enumerated $j=$ $1,2, \ldots, N$. We use $N=10^{4}$ particles in our simulations. Using the conserved quantity $x^{j}-$

$m_{e} v_{y}^{j} / e B_{0}=x_{0}^{j}$, where $x_{0}^{j}$ is an integration constant (we choose $x_{0}^{j}=0$ here), $x^{j}$ can be eliminated and the system can be normalized and rewritten in the dimensionless form

$$
\frac{d u_{x}^{j}}{d t}=-A \sin \left(u_{y}^{j}-\Omega t\right)-u_{y}^{j}
$$




$$
\frac{d u_{y}^{j}}{d t}=u_{x}^{j}
$$

where $A=e k E_{0} / m_{e} \omega_{c e}^{2}$ is the amplitude and $\Omega=\omega / \omega_{c e}$ the frequency of the electrostatic wave. Here the velocities are normalized by $\omega_{c e} / k$ and time is normalized by $\omega_{c e}^{-1}$.

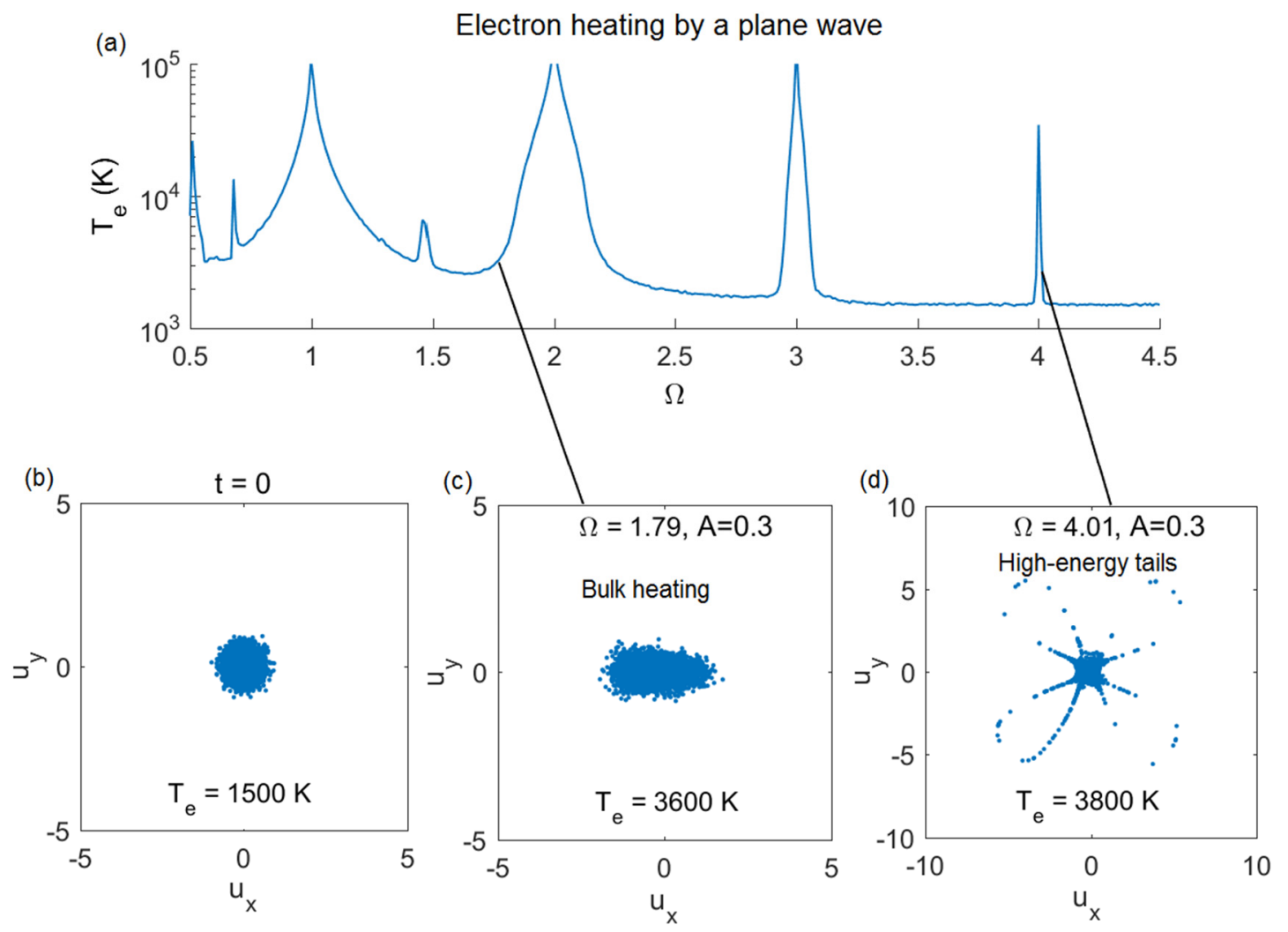

FIG. 10. The electron heating by a plane electrostatic wave, showing a) the kinetic electron temperature $T_{e}$ for electrons subject to a wave with different normalized wave frequencies $\Omega=$ $\omega / \omega_{c e}$, with wavenumber $k=15 \mathrm{~m}^{-1}$, wave electric field $E_{0}=9.4 \mathrm{~V} / \mathrm{m}$, and background magnetic field $B_{0}=5.17 \times 10^{-5} \mathrm{~T}$, giving $A=0.3$. (b) The electron distribution is initially Maxwellian with the temperature $T_{e}=1500 \mathrm{~K}$. (c) Bulk heating occurs between resonances, here at $\Omega=1.79$ corresponding to Peak 03 at $2.6 \mathrm{MHz}$ in Fig. 2 b. (d) Resonant acceleration occur near cyclotron resonances (integer values of $\Omega$ ) leading to high-energy tails, for $\Omega=4.01$, corresponding to the wave frequency of a mode-converted UH wave for the case $\omega_{0}=4.01 \omega_{c e}$. 
Model equations similar to Eqs. (11) and (12) have been used in the past to study stochastic heating in various plasma systems. Karney (1978) gives the condition for stochastic motion $E_{0} / B_{0}>(1 / 4)\left(\omega_{c e} / \omega\right)^{1 / 3}\left(\omega / k_{\perp}\right)$ for particles with $v>\omega / k$. For $v<\omega / k$ and $\Omega<1$ McChesney et al. (1987) give the condition for stochasticity $A \gtrsim 1$, which also has been obtained in the context of stochastic electron heating by shocks Balikhin et al., (1993). Most prominently, when charged particles are accelerated by electrostatic waves near cyclotron harmonics Fukuyama et al. (1977) the trajectories of the particles can become unstable when their initial speeds are higher than the phase speed of the wave, leading to stochastic motion of the particles and high-energy tails in the particle distribution function.

Figure 10 shows the result of a set of simulations over 1000 wave periods where the electrons are initially Maxwell distributed (cf. Fig. 10b) with the temperature $T_{e 0}=1500 \mathrm{~K}$. The wavenumber is chosen to be $k=15 \mathrm{~m}^{-1}$, and the ratio $\Omega=\omega / \omega_{c e}$ is varied in steps, keeping $\omega_{c e}=9.09 \times 10^{6} s^{-1}(1.45 \mathrm{MHz})$ fixed. The wave electric field amplitude is $E_{0}=9.4 \mathrm{~V} / \mathrm{m}$, corresponding to the normalized amplitude $A=0.3$. The kinetic electron "temperature" is calculated from the variance of the test particle velocities, as

$$
T_{e}=\frac{m_{e}}{2 k_{B}}\left[\frac{1}{N} \sum_{j}\left(\left(v_{x}^{j}\right)^{2}+\left(v_{y}^{j}\right)^{2}\right)-\bar{v}_{x}^{2}-\bar{v}_{y}^{2}\right]
$$

where $\bar{v}_{x}=\frac{1}{N} \sum_{j} v_{x}^{j}$ and $\bar{v}_{y}=\frac{1}{N} \sum_{j} v_{y}^{j}$ are the mean velocity components. The kinetic electron temperature coincides with the conventional temperature for a Maxwell distribution, and is a measure of the mean kinetic energy for a distribution function having suprathermal tails. One can see significant increases of the kinetic electron temperature for wave frequencies near the electron cyclotron harmonics corresponding to integer values of $\Omega$. For higher frequencies, the regions of strong heating occur in narrower regions around the harmonics. It turns out bulk heating takes place when $\Omega$ is between harmonics, such as $\Omega=1.76$ in Fig. 10c. On the other hand, near cyclotron harmonics, such as $\Omega=4.01$ in Fig. 10d, a small number of test particles are accelerated to very high velocities in a flower-like pattern in velocity space. The particle trajectories along the edges of these leave patterns will give rise to the "kicks" of acceleration and deceleration seen in the single particle simulation in Fig. 7d. In this case the electron distribution function becomes strongly non-Maxwellian, with high-energy tails, and the concept of "temperature" breaks down. 
The results are consistent with Fig. 6b, where a few accelerated teste particles were observed when the pump frequency was taken to be $\omega_{0}=4.01 \omega_{c e}$.

\section{SUMMARY AND CONCLUSIONS}

We have presented the results of Vlasov and test-particle simulations that indicate that UH/EB energization of electrons inside density cavities changes from bulk heating to a resonant acceleration that create suprathermal tails when the pump frequency approaches the $4^{\text {th }}$ electron cyclotron harmonic. We found that each type of heating is associated with a different set of waves. The bulk heating is driven by EB waves near $2.6 \mathrm{MHz}$, and not directly by UH waves even though the EB waves are nearly $20 \mathrm{~dB}$ lower in amplitude. The tail heating is due to resonant acceleration caused by UH waves if they are within $10-20 \mathrm{kHz}$ of the $4^{\text {th }}$ electron cyclotron harmonic. This result is consistent with previous theoretical and numerical studies of stochastic heating of charged particles by electrostatic waves near cyclotron harmonics Fukuyama et al. (1977), Grach (1999). The resonant electron acceleration is relevant to ionospheric artificial ionization experiments, where the creation of new plasma and formation of descending artificial ionospheric layers are observed when the pump frequency is tuned near one of the electron cyclotron harmonics Mishin and Pedersen (2011), Sergeev et al. (2013), Bernhardt et al. (2016). A comprehensive study that incorporates the results of the present paper to a simulation code similar to the one used in Eliasson et al. (2012) will be presented in a future publication.

Acknowledgments This research is supported by AFOSR grant F9550-13-1-0194 and NSF grant AGS 1158206. B.E. gratefully acknowledges support from the EPSRC (UK) grant EP/M009386/1. The authors are grateful to Paul Bernhardt, Carl Siefring and Stan Briczinski for providing SEE data. The raw data for this paper are available for download via FTP at http://ftp.astro.umd.edu/pub/SPP/GRL SSS paper data/. Amir C. Najmi (amir.c.najmi@gmail.com) may be contacted directly for access to either the whole data set or subsets of interest.

\section{References}

Balikhin M., Gedalin M., and Petrukovich A., "New mechanism for electron heating in shocks," Phys. Rev. Lett. 70, 1259-1263, doi:10.1103/PhysRevLett.70.1259 (1993).

Bernhardt, P. A., Siefring, C. L., Briczinski, S. J., McCarrick, M., and Michell, R. G., "Large ionospheric disturbances produced by the HAARP HF facility," Radio Sci. 51, 1081-1093, doi:10.1002/2015RS005883 (2016). 
Dysthe, K. B., Mjølhus, E., Pécseli, H. L., and Rypdal, K., “Thermal cavitons,” Phys. Scr., T2/2, 548-559, doi:10.1088/0031-8949/1982/T2B/040 (1982).

Eliasson, B., "Numerical simulations of the Fourier transformed Vlasov-Maxwell system in higher dimensions - theory and applications," Transp. Theor. Stat. Phys., 39(5), 387-465, doi:10.1080/00411450.2011.563711 (2010).

Eliasson, B., Shao X., Milikh, G., Mishin, E. V., and Papadopoulos, K., "Numerical modeling of artificial ionospheric layers driven by high-power HF heating," J. Geophys. Res. 117, A10321, doi:10.1029/2012JA018105 (2012).

Eliasson, B., and Papadopoulos, K., "Numerical study of anomalous absorption of O mode waves on magnetic field aligned striations," Geophys. Res. Lett. 42, 2603-2611, doi:10.1002/2015GL063751 (2015).

Fukuyama, A., Momota, H., Itatani,R., and Takizuka, T., "Stochastic acceleration by an electrostatic wave near ion cyclotron harmonics," Phys. Rev. Lett. 38, 701-705, doi:10.1103/PhysRevLett.38.701 (1977).

Grach, S. M. (1999), “On kinetic effects in the ionospheric F-region modified by powerful radio waves," Radiophys. Quantum. Electron., 42, 572-588.

Grach, S. M., Sergeev, E. N., Mishin, E.V., and Shindin, A. V., "Dynamic properties of ionospheric plasma turbulence, driven by high-power high-frequency radiowaves," PhysicsUspekhi 59(11), 1091-1128 (2016).

Gurevich, A. V., Zybin, K. P., and Lukyanov, A. V., "Stationary striations developed in the ionospheric modification,” Phys. Rev. Lett. 75, 2622-2625, doi:10.1103/PhysRevLett.75.2622 (1995).

Gurevich, A. V., "Nonlinear effects in the ionosphere," Phys. Usp. 50(11), 1091-1121, doi: 10.1070/PU2007v050n11ABEH006212 (2007).

Huang J. and Kuo, S. P., "A theoretical model for the broad upshifted maximum in the stimulated electromagnetic emission spectrum,” J. Geophys. Res., 99(A10), 19569-19576, doi:10.1029/94JA01261 (1994). 
Jones, T. B., Robinson, T. R., Stubbe, P., and Kopka, H., "Frequency dependence of anomalous absorption caused by high power radio waves,” J. Atmos. Terr. Phys. 46(2), 147-153 (1984).

Karney, C. F. F., "Stochastic ion heating by a lower hybrid wave," Phys. Fluids 21, 1584-1599, doi:10.1063/1.862406 (1978).

Lominadze, D. G., “Cyclotron Waves in Plasmas” (Pergamon Press, New York, 1981).

McChesney, J., Stern, R. A., and Bellan, P., "Observation of fast stochastic ion heating by drift waves,” Phys. Rev. Lett. 59, 1436, doi:10.1103/PhysRevLett.59.1436 (1987).

Mishin, E. N., Burke, W. J., and Pedersen T., "On the onset of HF-induced airglow at HAARP," J. Geophys. Res., 109, A02305, doi:10.1029/2003JA010205 (2004).

Mishin, E. and Pedersen, T., "Ionizing wave via high-power HF acceleration," Geophys. Res. Lett., 38, L01105, doi:10.1029/2010GL046045 (2011).

Mishin, E., Watkins, B., Lehtinen, N., Eliasson, B., Pedersen, T., and Grach, S., “Artificial ionospheric layers driven by high-frequency radiowaves: An assessment," J. Geophys. Res. Space Phys. 121, 3497-3524, doi:10.1002/2015JA021823 (2016).

Mjølhus, E., "Anomalous absorption and reflection in ionospheric radio modification experiments,” J. Geophys. Res. 90(A5), 4269-4279, doi:10.1029/JA090iA05p04269 (1985).

Mjølhus, E., "Theoretical model for long time stimulated electromagnetic emission generation in ionospheric radio modification experiments," J. Geophys. Res. 103(A7), 14711-14729, doi:10.1029/98JA00927 (1988).

Najmi, A., Milikh, G., Secan, G., Chiang, K., Psiaki, M., Bernhardt, P., Briczinski, S., Siefring, C., Chang, C. L., and Papadopoulos, K., "Generation and detection of super small striations by F region HF heating," J. Geophys. Res. Space Phys. 119, 6000-6010, doi:10.1002/2014JA020038 (2014).

Najmi, A., Eliasson, B., Shao, X., Milikh, G., and Papadopoulos, K., "Simulations of ionospheric turbulence produced by HF heating near the upper hybrid layer," Radio Sci. 51(6), 704-717, doi:10.1002/2015 RS005866 (2016). 
Papadopoulos, K., Eliasson, B., Chang, C. L., Shao, X., Milikh G., and Watkins, B. "New Results on Artificial Plasma Layers" Invited Presentation, $19^{\text {th }}$ Ionospheric Interactions Workshop, Aprril 23, 2013, Arecibo, PR.

Pedersen, T., Gustavsson, B., Mishin, E., MacKenzie, E., Carlson, H. C., Starks, H. C., and Mills, T., "Optical ring formation and ionization production in high-power HF heating experiments at HAARP,” Geophys. Res. Lett. 36, L18107, doi:10.1029/2009GL040047 (2009).

Pedersen, T., Gustavsson, B., Mishin, E., Kendall, E., Mills, T., Carlson, H. C., and Snyder, A. L., "Creation of artificial ionospheric layers using high-power HF waves," Geophys. Res. Lett. 37(2), L02106, doi:10.1029/2009GL041895 (2010).

Sergeev, E., Grach, S., Shindin, A., Mishin, E., Bernhardt, P., Briczinski, S., Isham, B., Broughton, M., LaBelle, J., and Watkins, W., "Artificial ionospheric layers during pump frequency stepping near the 4th gyroharmonic at HAARP," Phys. Rev. Lett. 110, 06500, doi:10.1103/PhysRevLett.110.065002 (2013).

Shevchenko, V., Cunningham, G., . Gurchenko, A., Gusakov, E., Lloyd, B., O’Brien, M., Saveliev, A., Surkov, A., Volpe, F., and Walsh, M., "Development of electron Bernstein wave research in MAST," Fusion Sci. Technol. 52(2), 202-215 (2007).

Stenzel, R. L., and Gould, R. W., "Upper-hybrid resonance absorption, emission and heating of an afterglow plasma column,” J. Appl. Phys. 42, 4225-4235 (1971).

Timofeev, A. V., "Cyclotron oscillations of an equilibrium plasma," in B. B. Kadomtsev (ed.) Reviews of Plasma Physics 14, 63-252 (Consultants Bureau, New York, 1989).

Xi, H., and Scales, W. A., "Numerical simulation studies on the broad upshifted maximum of ionospheric stimulated electromagnetic emission,” J. Geophys. Res. 106(A7), 12787-12801, doi:10.1029/2000JA000322 (2001). 